\title{
Impact of Photon Noise on the Reliability of a Motion- Sensitive Neuron in the Fly's Visual System
}

\author{
Jan Grewe, ${ }^{1}$ Jutta Kretzberg, ${ }^{2}$ Anne-Kathrin Warzecha, ${ }^{1}$ and Martin Egelhaaf ${ }^{1}$ \\ ${ }^{1}$ Department of Neurobiology, Universität Bielefeld, 33501 Bielefeld, Germany, and ${ }^{2}$ Salk Institute-Computational Neurobiology Laboratory, La Jolla, \\ California 92037-1099
}

\begin{abstract}
Variable behavioral responses to identical visual stimuli can, in part, be traced back to variable neuronal signals that provide unreliable information about the outside world. This unreliability in encoding of visual information is caused by several noise sources such as photon noise, synaptic noise, or the stochastic nature of ion channels. Neurons of the fly's visual motion pathway have been claimed to represent perfect encoders, with photon noise as the main noise source limiting their performance. Other studies on the fly's visual system suggest, however, that internal noise emerging within the nervous system also affects the reliability of motion vision. To resolve these contradictory interpretations, we performed an electrophysiological investigation, inspired by the "equivalent noise" paradigm applied in psychophysics, on the fly's motion-sensitive $\mathrm{H} 1$ neuron. Noise-like brightness fluctuations of different strength were superimposed on the motion stimuli. Because the noise level found to affect the temporal properties of the spike responses is much larger than the estimate of photon noise under the experimental conditions, our results indicate that motion vision is more likely to be limited by internal sources of variability than by photon noise.
\end{abstract}

Key words: insect vision; reliability; photon noise; motion vision; equivalent noise; visual motion processing

\section{Introduction}

Animals, including humans, respond to sensory stimuli with limited reliability. In the brain, this behavioral or perceptual variability is reflected in variable neuronal responses providing unreliable information about the outside world. For instance, neurons in the visual cortex of cats and monkeys respond to stimuli with a spike count variance in the range of the mean activity (Tolhurst et al.,1983; Vogels et al.,1989; Britten et al.,1993; Barberini et al.,2000). Although neuronal variability of insect visual interneurons (Warzecha and Egelhaaf, 1999, 2001; Warzecha et al., 2000; de Ruyter van Steveninck et al., 2001; Borst and Haag, 2002) and of vertebrate retinal ganglion cells is somewhat smaller (Levine et al., 1988; Berry et al., 1997; Kara et al., 2000), it still limits the reliability of behavioral reactions.

Various noise sources within the nervous system constrain the reliability of neuronal responses, such as the phototransduction process (Rodieck, 1998), the stochastic nature of ion channels, as well as synaptic transmission (Johnston and Wu, 1995). In addition, the incoming visual signal is inherently noisy because of the quantum nature of light (Rodieck, 1998). Especially at extremely low light levels, the absorption of single photons triggers prominent membrane potential changes in the photoreceptor [insects (Hardie, 1979); vertebrates (Rodieck,1998)]. In flies, single-

\footnotetext{
Received May 8, 2003; revised Sept. 9, 2003; accepted Sept. 12, 2003

This work was supported by the Deutsche Forschungsgemeinschaft. We thank G. Schwerdtfeger, who performed part of the experiments. We are grateful to N. Boeddeker, J. P. Lindemann, K. Meyer, F. Oddos, K. Karmeier, and R. Kurtz for helpful comments on this manuscript.

Correspondence should be addressed to Jan Grewe, Lehrstuhl für Neurobiologie, Universität Bielefeld, Universitätsstrasse 25, Postfach 100131, 33501 Bielefeld, Germany. E-mail: jan.grewe@uni-bielefeld.de. Copyright $\odot 2003$ Society for Neuroscience $\quad$ 0270-6474/03/2310776-08\$15.00/0
}

photon effects could then be detected in spike patterns of the motion-sensitive $\mathrm{H} 1$ neuron that is several synapses away from the photoreceptors (Lillywhite and Dvorak, 1981) and in behavioral responses (Reichardt, 1965). In humans, single-photon absorptions were found to affect perception (Bouman et al., 1985). Thus, at the sensitivity threshold, the reliability of motion vision is limited by photon noise.

Although individual photons contribute much less to the overall photoreceptor response at higher light levels, it was concluded that, even in daylight, photon noise is the major source of variability in the fly motion-sensitive $\mathrm{H} 1$ neuron (de Ruyter van Steveninck and Bialek, 1995; Borst and Haag, 2001; Lewen et al., 2001). The $\mathrm{H} 1$ neuron is a well established system for analyzing the reliability of neural coding (for review, see Egelhaaf and Warzecha, 1999; de Ruyter van Steveninck, et al., 2001; Warzecha and Egelhaaf, 2001; Egelhaaf et al., 2002; Haag and Borst, 2002). All internal sources of variability were claimed to be of minor relevance, implying that neuronal information processing is virtually perfect (de Ruyter van Steveninck and Bialek, 1995; Borst and Haag, 2001; Lewen et al., 2001). However, at least for the lightadapted eye, noise sources inherent in synaptic transmission between photoreceptors and second-order neurons were shown to significantly affect the reliability with which visual information is signaled to higher-order processing stages (Laughlin et al., 1987; de Ruyter van Steveninck and Laughlin, 1996).

Is response reliability of $\mathrm{H} 1$ limited by photon noise or also shaped by noise sources inherent in the nervous system? In an experimental design adapted from psychophysics, external noise was introduced into the system. Random brightness modulations, which corrupt the input signal similar to photon noise, were superimposed on random dots, moving in the preferred 
direction of H1. We examined how much noise could be added before the motion response was affected noticeably and how this noise level relates to photon noise under the experimental conditions.

\section{Materials and Methods}

Electrophysiology. Experiments were performed on 21 female blowflies (Lucilia species), 2-8 d old, at temperatures between $22^{\circ}$ and $26^{\circ} \mathrm{C}$. The animals were obtained from laboratory stocks. Dissection was performed as described previously (Warzecha et al., 1993). After dissection, the flies were adjusted in the experimental setup with respect to the symmetry of the deep pseudopupil (Franceschini, 1975).

The spike activity of the $\mathrm{H} 1$ cell, a directionally selective motionsensitive neuron (Hausen, 1976) was recorded extracellulary at its output region in the left half of the fly's visual system. This $\mathrm{H} 1$ cell has its input region in the right visual field and conveys visual motion information to the contralateral visual system. To reduce background noise resulting from the activity of neurons that receive input from the left visual field, the left eye was covered with black varnish. Insulated tungsten electrodes were used for recording. As reference electrode, a glass capillary filled with Ringer's solution (Hausen, 1982) was used. The recorded signals were amplified (factor 3000) and bandpass filtered (corner frequencies, $300 \mathrm{~Hz}$ and $3 \mathrm{kHz}$ ). Recorded action potentials were transformed into pulses of fixed height and duration, fed into a personal computer, and stored onto hard disk for off-line analysis. Data acquisition was performed at a sampling frequency of $1200 \mathrm{~Hz}$ using the analog input of a visual stimulus generator (VSG) 2/3 card (Cambridge Research Systems, Cambridge, UK). Data were analyzed using Matlab (version 6.5, release 13; The Mathworks Inc.), and some routines were written in ANSI-C.

Visual stimulation. Visual stimuli were presented to the fly at $300 \mathrm{~Hz}$ with a Joyce Scope DM5 monochrome (P-31 phosphor; decay to $10 \%$ in $\sim 40 \mu \mathrm{sec}$ ) computer monitor (Joyce Electronics, Cambridge, UK). The brightness range of the monitor was resolved with eight bits. The monitor was controlled using the VSG 2/3 graphics card (Cambridge Research Systems). The card was programmed using Borland Delphi 3.0 and VSG Software Library version 6.0.

As seen by the fly, the monitor screen $(464 \times 375$ pixel resolution $)$ had a horizontal and vertical extent of $127^{\circ}$ and $120^{\circ}$, respectively. The screen center was positioned at $0^{\circ}$ elevation and $20^{\circ}$ azimuth. At this point, one pixel had a vertical and horizontal extent of $\sim 0.5^{\circ}$.

Although the frame rate was $300 \mathrm{~Hz}$, the update rate of the presented motion stimulus was $150 \mathrm{~Hz}$. Cross-correlation of 200 response traces of $\mathrm{H} 1$ revealed no time locking to either of these frequencies.

Random dot stimuli. Random dot kinematograms were used as visual stimuli. They consisted of 10 bright dots with a vertical and horizontal extent of $\sim 1.5^{\circ}$, as seen by the fly. In the first frame of the stimulus sequence, the dots were positioned randomly in front of a dark background. In subsequent frames, these dots were moved in the preferred direction of the $\mathrm{H} 1$ cell [i.e., from back to front, by steps of three pixels (corresponding to $1.5^{\circ}$ ) per frame]. The position of the dots was updated at a rate of $150 \mathrm{~Hz}$, which results in a velocity of $\sim 450^{\circ}$ /sec in the screen center. The same dot pattern was used in every stimulus presentation. The stimulation sequence consisted of a $3 \mathrm{sec}$ motion and $7 \mathrm{sec}$ interstimulus interval, during which the monitor showed the starting image of the motion sequence. At mean brightness, all dots had a radiance of $2.7 \times$ $10^{-1} \mathrm{~W} \times \mathrm{m}^{-2} \times \mathrm{sr}^{-1}$ and a background of $3.0 \times 10^{-5} \mathrm{~W} \times \mathrm{m}^{-2} \times$ $\mathrm{sr}^{-1}$ (corresponding to $130 \mathrm{~cd} / \mathrm{m}^{2}$ and $0.001 \mathrm{~cd} / \mathrm{m}^{2}$, respectively). We indicate light intensities and intensity changes in relative units. Because the brightness range of our stimulus was resolved with eight bits (i.e., 256 intensity levels), an intensity level of 127 corresponds to the mean brightness of the dots and 0 to the background brightness. The gamma correction of the video screen was approved to result in a virtually linear relationship between intensity level and real brightness.

To introduce external noise into the fly's visual system, random brightness modulations were superimposed on the dots (i.e., the brightness of each dot was varied between subsequent frames). As shown in Figure 1, the brightness values were taken randomly from Gaussian distributions with the same mean but different SDs. The distributions were

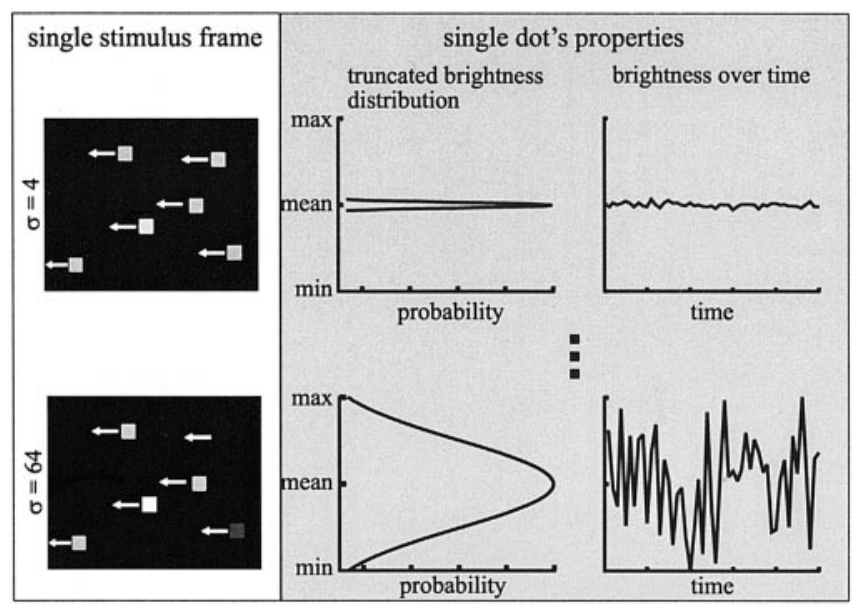

Figure 1. Visual stimuli for the smallest ( $\sigma=4$; top) and the largest ( $\sigma=64$; bottom) noise level. On the left, the same frame of the motion sequence is shown. For the first frame, a number of dots are placed randomly on the screen, and in subsequent frames the dots are shifted in the preferred direction of the $\mathrm{H} 1$ cell (indicated by the arrows). As shown, each dot has its own brightness. Properties of a sample dot are shown on the right. The noise sequence applied to this dot during presentation was made up of randomly drawn brightness values drawn from a Gaussian distribution centered at the mean brightness (127 on the eight-bit scale) and a SD of $\sigma=4,8,16,32$, or 64, defining the noise level (shown for $\sigma=4$ and 64). This distribution was truncated at mean $\pm 2 \sigma$. Although the brightness variations over time are quite small (top), modulations covering the full brightness range are possible at the largest noise level $(\sigma=64)$.

truncated at mean level $\pm 2 \sigma$. We define the noise level as the SD of the original Gaussian. Five noise levels were used in the experiments $(\sigma=4$, $8,16,32$, and 64 brightness levels on the eight-bit scale). Thus, at the maximum noise level, brightness modulations covered the available brightness range entirely (Fig. 1, compare bottom).

In a first set of experiments, the time-dependent noise sequences added to all 10 dots were statistically independent. In a second set of experiments, the noise added on fractions of the dots was correlated. We used six correlation levels $(0,20,40,60,80$, and $100 \%)$ at two different noise levels ( $\sigma=16$ and $\sigma=64)$.

In both sets of experiments, the noise was added in two different ways: (1) the same brightness noise sequence was superimposed on the dots in each stimulus presentation ("reference stimulus"); and (2) different brightness noise sequences, yet with the same statistical properties, were used in each presentation ("test stimulus").

Experimental protocol. Before data acquisition, the fly was adapted to the stimulus and laboratory conditions. For this purpose, the stimulus with a randomly chosen noise level was presented 40 times in a row, which lasted $\sim 7 \mathrm{~min}$.

After this adaptation period, the experiments were performed blockwise for each noise level $(\sigma=4,8,16,32$, and 64) or each correlation level $(0,20,40,60,80$, or $100 \%)$. The interstimulus interval, during which the monitor showed the starting image of the motion sequence with all dots at mean brightness, was used for data storage and for providing Ringer's solution to the fly, if necessary. Both reference and test stimuli were presented 30 times in random order within each noise level/correlation block. The blocks were also shuffled in experiments performed on different $\mathrm{H} 1$ cells in different animals.

For additional analysis, 25 of the 30 responses to each stimulus type were used. We selected those 25 spike trains that deviate least with respect to their mean activity from the mean response averaged over all reference and test responses.

Data analysis and comparison of spike trains. The aim of the experiments was to find the level of external noise that leads to a major change in the responses of the cell to the test stimuli, compared with the responses to the reference stimulus. Because reference and test stimuli have the same statistical properties, we expected them to lead to the same spike rate but possibly to different spike patterns. We applied two approaches to detect differences in the temporal properties of the spike trains. 
The first approach compares spike trains by calculating the minimal costs of transforming one spike train into another (Victor and Purpura, 1997). The transformation is performed by deleting, inserting, or temporally shifting the single spikes. Each of these procedures is linked to defined costs. Deleting or inserting the single spikes has the cost of "1." The costs for a temporal shift ("q" per second) is variable and determines the temporal resolution of the measure. For example, a q value of 200 equals a temporal resolution of 10 msec: for this time scale, spikes in two response traces are considered "nearly coincident" if a given spike in one of the spike trains is shifted by less than $\pm 10 \mathrm{msec}$, with respect to the corresponding spike in the other spike train. As long as this condition is met, it is "cheaper" to temporally adjust the spikes in the two spike trains by shifting than by deleting and inserting one of them. In our analysis, we used a set of nine different values for $\mathrm{q}(\mathrm{q}=2,4,8,16,32,64,128,256$, and 512) that correspond to temporal resolutions of $1000,500,250,125,62.5,31.25,15.625,7.8125$, and 3.90625 msec. Moreover, we calculated the similarity between test and reference responses on the basis of the mean spike rate over the entire stimulation interval (see Figs. $4 b, 5 b$,e, rightmost data points).

The second approach calculates the distance between two spike trains by determining spikecount differences between the smoothed spike trains (Kretzberg et al., 2001):

$$
\operatorname{dist}\left(X^{\tau}, Y^{\tau}\right)=\sqrt{\frac{1}{N} \sum_{j=1}^{N}\left(X_{j}^{\tau}-Y_{j}^{\tau}\right)^{2}}
$$

with $N$ as the number of time steps, $\tau$ as filter width (milliseconds), and $X^{\tau}, Y^{\tau}$ as the smoothed spike trains that are to be compared. A rectangular filter of variable width was used for smoothing. We calculated the distances using six different filter widths $(500,200,100,50$, 20 , and $10 \mathrm{msec}$ ).

Classification of responses. To determine the similarity of the spike trains, we took each single reference response (e.g., the highlighted spike train in Fig. 2a) and computed the mean similarity (see above) to the other reference responses and to the test responses. We can assume the reference responses to be more similar to each other than to the test responses if the external noise affects the responses. Thus, a reference response is subsequently classified correctly as a reference response when its similarity within the reference responses is higher than between reference and test responses. This procedure was performed for all 25 reference responses. The percentage of correct classifications represents a measure of the discriminability of reference and test responses. If the test responses do not differ more from the reference responses than the reference responses do from each other, the percentage of correct assignments is $50 \%$. In this case, the added noise does not affect detectably the temporal structure of the spike trains, and their variability is dominated by noise sources intrinsic to the nervous system, by photon noise, or by both noise sources. If both types of responses can be discriminated perfectly, the percentage of correct classifications is $100 \%$. In this case, the responses are affected heavily by the external noise that was added to the stimuli. We assume a major influence of the external noise on the responses when the discrimination performance exceeds $75 \%$, a threshold value often used in psychophysics.

Domain of uncertainty. To assess whether deviations of the discrimination performance from chance level (Fig. 2, compare $b, c$ ) are attribut- able to the external noise, we calculated the percentage of correct decisions for 10 different sets of shuffled responses [i.e., we replaced half of the reference responses (selected by chance) with the same number of test responses chosen randomly]. For each shuffle, the classification performance was calculated as described above. A $50 \%$ performance is expected, because the shuffle consists of responses of either type mixed in equal shares. A deviation from chance level can, thus, be understood as a measure of variability caused by any kind of noise. The shaded area in Figure 2, $b$ and $c$, shows the "domain of uncertainty" that is defined by the mean (dotted line) and twice the SD of the classification performances obtained by comparing the 10 different sets of shuffled responses. Classification performances that fall into the domain of uncertainty are likely to be a result of the noise in the system (including photon noise) and not of the added external noise.

Estimation of photon noise. Following the approach of de Ruyter van Steveninck (1986), the number of photons impinging per second on a single photoreceptor can be estimated according to:

$$
N=\epsilon_{\mathrm{sp}} \times \int_{0}^{\infty} L_{\mathrm{ph}}(\lambda) \times S_{\mathrm{pr}}(\lambda) d \lambda
$$


with $\lambda$ as the wavelength, $\epsilon_{\mathrm{sp}}\left(\mathrm{m}^{2} \times \mathrm{sr}\right)$ as the spatial efficiency factor, $L_{\mathrm{ph}}(\lambda)$ (photons $\times \mathrm{m}^{-2} \times \mathrm{sr}^{-1} \times \mathrm{sec}^{-1} \times \mathrm{nm}^{-1}$ ) as the spectral density of the photon flux, and $S_{p r}(\lambda)$ as the relative spectral sensitivity of fly photoreceptors (type R1-6). The spatial efficiency factor is given by the product of lens surface and photoreceptor solid angle, which results in:

$$
\epsilon_{\mathrm{sp}}=3.5 \times 10^{-13} \mathrm{~m}^{2} \times \mathrm{sr}
$$

assuming a circular lens (van Hateren, 1984) with a point spread function approximation as a circular symmetric Gaussian (Götz, 1965). The lens diameter and the acceptance angle are assumed as $\mathrm{D}=30 \mu \mathrm{m}$ and $\Delta \rho=$ $1.2^{\circ}$, respectively (Smakman et al., 1984). The integral in formula 2 is the spectral overlap $L_{s o}$, which can be calculated for the mean radiance of the dots of $2.7 \times 10^{-1} \mathrm{~W} \times \mathrm{m}^{-2} \times \mathrm{sr}^{-1}$ to (de Ruyter van Steveninck, 1986):

$$
L=4.47 \times 10^{17} \text { photons } \times \mathrm{m}^{-2} \times \mathrm{sr}^{-1} \times \mathrm{sec}^{-1}
$$

Inserting formulas 3 and 4 in formula 2 we get:

$$
N=L_{\mathrm{so}} \times \epsilon_{\mathrm{sp}}=1.56 \times 10^{5} \text { photons } \times \mathrm{sec}^{-1}
$$

Thus, $\sim 1034$ photons impinge on a photoreceptor while a dot is in its receptive field $(1 / 150 \mathrm{sec})$.

Because the number of photon arrivals is a statistical event following the Poisson distribution, the variance of which is equal to the mean, the $\mathrm{SD}$ and, thus, the signal-to-noise ratio (SNR) amounts to $\sim 32$. To mimic an SNR matching photon noise in our stimulation paradigm, brightness noise with a SD of $\sigma=4$ [with a mean brightness of 127 on the eight-bit scale $(0 \ldots 255)]$ would have to be added to the moving dots (i.e., $128 / 4=32$ ).

Persistence of the $\mathrm{P}-31$ phosphor increases the time a dot stimulates a photoreceptor and, thus, slightly increases the number of photons impinging on the receptors. Consequently, photon noise is slightly overestimated by the above approximation.

\section{Results}

To find out whether the reliability of $\mathrm{H} 1$ responses to motion stimuli is limited by photon noise or noise sources intrinsic to the nervous system, the consequences of external brightness noise superimposed on a random dot kinematogram were analyzed. The brightness of the 10 dots moving coherently in the preferred direction of the $\mathrm{H} 1$ cell was modulated in two ways. The timedependent brightness noise was either exactly the same in each stimulus presentation (reference stimulus) or it was different but had the same statistical properties in each presentation (test stimulus). We examined for a wide range of time scales to what extent the temporal structure of spike responses was affected by the added noise.

In case of photon noise being the dominant noise source limiting motion vision, the added noise, as soon as it is larger than photon noise itself, should affect the responses. Thus, the reference responses should be more similar to each other than to the test responses. This is because the reference stimulus is, apart from photon noise, the same for each trial, and the variability in the reference responses reflects the consequences of noise sources in the nervous system and of photon noise. In contrast, the variability of the test responses is also affected by the external noise introduced by using a different stimulus for every presentation, unless the external noise level is negligible in the presence of the other noise sources that cannot be controlled by the experimenter. The larger the brightness noise level needed to have a major impact on the responses, the less important is photon noise for the reliability of motion vision in $\mathrm{H} 1$.

\section{Impact of uncorrelated noise}

Because the statistical properties of reference and test stimuli were identical, we expected them to elicit the same average re-

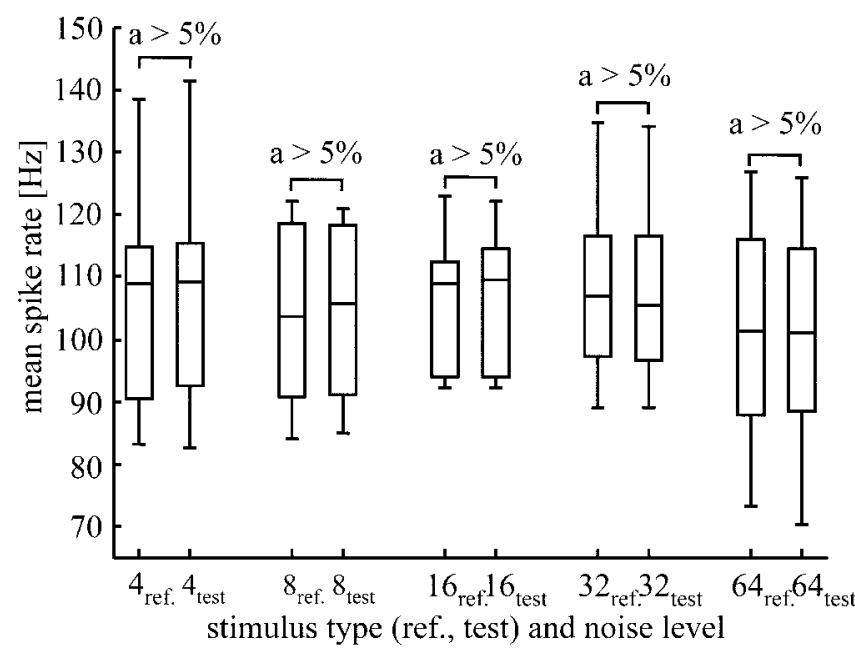

Figure 3. Mean firing rate of $\mathrm{H} 1$ in response to reference and test stimuli at the five noise levels $(\sigma=4,8,16,32$, and 64$)$ used in the experiments. The box and whisker diagrams represent the distribution of mean firing rates, averaged over all presentations of each stimulus type. All diagrams are based on the same $11 \mathrm{H} 1$ neurons recorded in 11 different flies. The horizontal lines in the boxes represent the lower, median, and upper quartile of the data. The whiskers extending vertically from the boxes show the extent of the rest of the data. The distributions were tested for significant differences using a Wilcoxon's test for paired samples. An $\alpha$ value, shown above each pair of distributions, larger than $5 \%$ denotes a nonsignificant difference.

sponses. Comparing the mean response amplitudes for all noise levels using a Wilcoxon's test for paired samples confirmed this expectation (Fig. 3). Thus, if there were differences in the responses to reference and test stimuli, they existed only on a finer time scale (i.e., in the temporal spike pattern). The raster plots shown in Figure $2 a$ reveal some variability in the spike trains, even if the responses were elicited by repeated presentation of the same stimulus (Fig. $2 a$; reference responses at maximum noise level). In case of different noise sequences superimposed on the moving dots at each presentation (Fig. $2 a$; test responses at maximum noise level), the responses appear not to be more variable than the reference responses. Under both stimulus conditions, a common time structure is visible despite differences in the timing of individual spikes. Based on the raster plots, it is hard to tell whether the individual spike trains obtained under the reference condition are more similar to each other than to spike trains obtained with the test stimulus. Therefore, we scrutinized the responses by quantitative measures.

Two different measures were applied to quantify the similarity of individual spike trains (see Materials and Methods). As shown for the single cell example in Figure 2, $b$ and Figure $c$ [measures developed by Victor and Purpura (1997) (Fig. $2 b$ ) and Kretzberg et al. (2001) (Fig. 2c) were used], the classification performance varies $\sim 50 \%$ for small noise levels; it increases with increasing noise level to almost perfect discrimination. The responses of this $\mathrm{H} 1$ cell to reference and test stimulus can be distinguished reliably at noise levels larger than $\sigma=32$ if the $75 \%$ criterion is satisfied and the data value is outside the domain of uncertainty. At maximum noise level, the responses can be distinguished perfectly. Because both measures of similarity lead to qualitatively the same results for all analyzed cells, only results obtained with the measure of Victor and Purpura (1997) will be shown below.

The average discrimination performance $(n=11 \mathrm{H} 1$ cells recorded in 11 different flies) is shown in Figure $4 a$ as a function of both the temporal resolution and the noise level. In some flies, 
the $75 \%$ criterion, as shown in Fig. $2 b$, was reached for high noise levels whereas other recordings revealed discrimination performances below this threshold, even at the highest noise levels used in the experiments. The averaged discrimination performance peaks at large noise levels and at temporal resolutions between 5 and 20 msec. Finer resolutions yield performances, even at the highest noise level, below threshold and only weakly above the domain of uncertainty (Fig. $4 b$ ). On average, temporal resolutions coarser than $62.5 \mathrm{msec}$ also result in no significant discrimination. Even for the temporal resolution at which the discrimination performance is best $(15.625 \mathrm{msec})$, the performance is significantly better than chance level only at noise levels above $\sigma=$ 32 (Fig. 4c). Hence, the temporal structure of the neuronal responses is affected systematically only at noise levels exceeding this value. However, even at the highest noise level the 75\% threshold is not reached, on average. In none of the recordings was the discrimination threshold reached with noise levels lower than $\sigma=$ 32. As is common in psychophysical experiments, this value may be understood as an estimate of the overall noise in the system, including internal sources of variability as well as photon noise (Pelli, 1990). In our stimulus paradigm, photon noise is estimated to equal a brightness modulation of approximately $\sigma=4$ (see Materials and Methods). As can be concluded from the experiments, this level of added noise has no major impact on the mean response amplitude and the temporal fine structure of the cell.

\section{Impact of correlated noise}

In the experiments described so far, the noise added to the different moving dots was uncorrelated. The effect of external noise on a neuron can be expected to increase if the noise carried by the different inputs of the neuron is correlated to some extent (Zohary et al., 1994). Because photon noise is uncorrelated at the different photoreceptors, the degree of correlation needed to affect the neuronal response pattern can be used as another way to assess the role of photon noise versus noise intrinsic to the nervous system for the reliability of visual motion computation. In a second set of experiments $(n=10$ recorded $\mathrm{H} 1$ cells in another set of 10 different flies), the noise superimposed on a fraction of the dots was correlated. The number of dots with correlated noise was $0,2,4$, 6,8 , or 10 of 10 dots. When the correlation at maximum noise level was increased, the $\mathrm{H} 1$ responses were affected not only on a

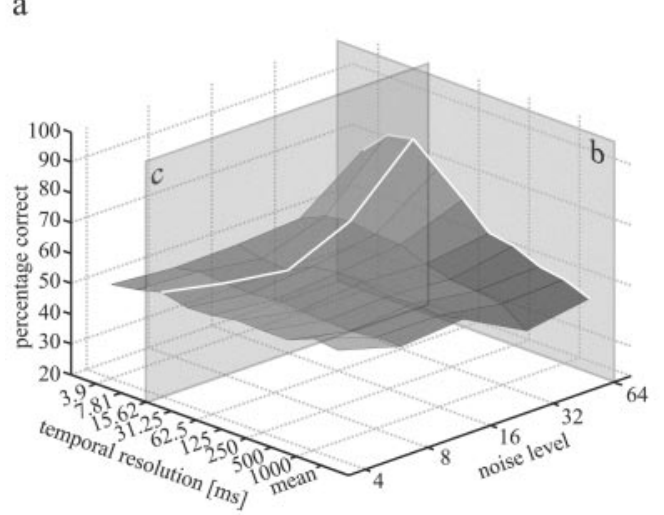

b

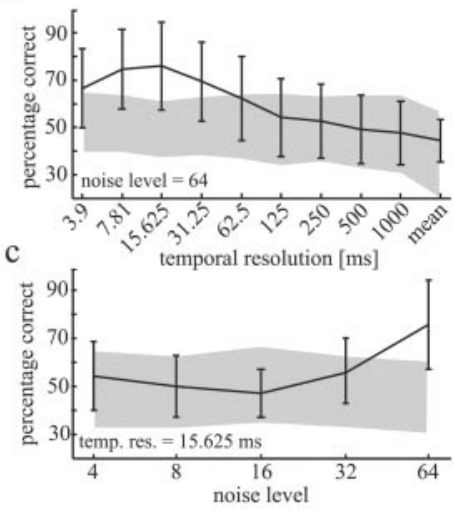

Figure 4. Averaged discrimination of reference and test responses as a function of the noise level added to the stimuli and the temporal resolution of the measure of discrimination as developed by Victor and Purpura (1997). Data are based on a set of $11 \mathrm{H} 1$ neurons recorded in 11 different flies. Transects $b$ and care shown in more detail in $b$ and $c . b$, Mean discrimination of reference and test responses at the maximum noise level $(\sigma=64)$ in dependence of the temporal resolution was used for data analysis. The error bars show the SD of percentage correct decisions across the 11 cells. The shaded area represents the domain of uncertainty (see Materials and Methods). c, As in b, but the noise amplitude was varied at the "best" temporal resolution (15.625 msec).

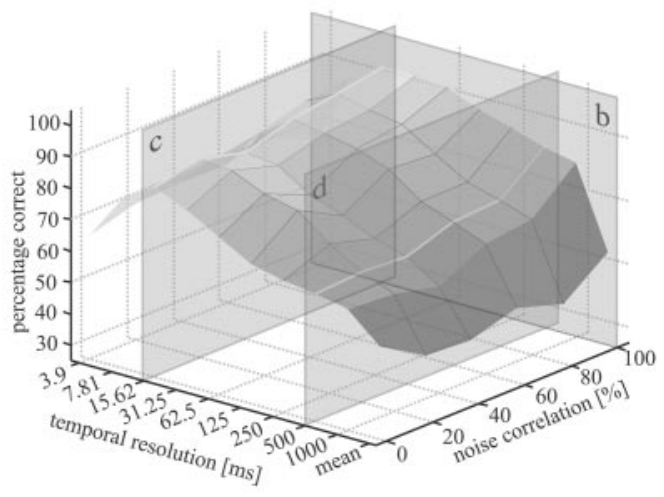

$\mathrm{e}$

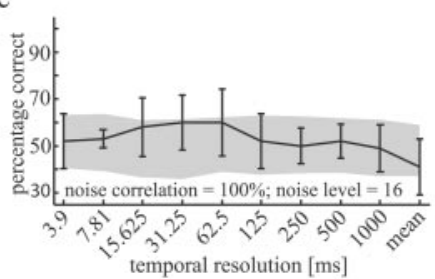

b
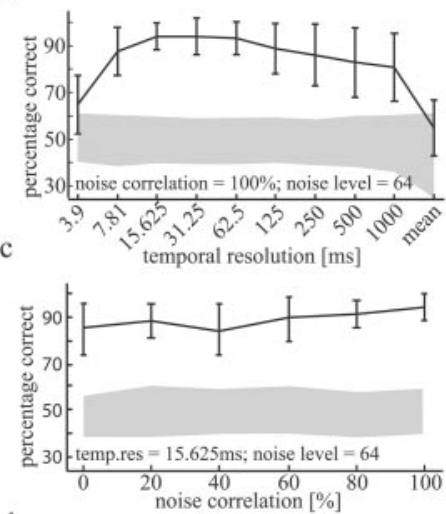

d



Figure 5. Mean discrimination performance as a function of the noise correlation and the temporal resolution of the measure of discrimination as developed by Victor and Purpura (1997). The experiments were performed at the maximum noise level $(\sigma=$ 64). The average performance is based on $10 \mathrm{H} 1$ recordings in another set of 10 different flies than those on which the data shown in Fig. 4 are based. The transections refer to sections shown in $b-d$. $b$, The average discrimination performance at $100 \%$ noise correlation expressed in percentage correct decisions as a function of the temporal resolution was used for data analysis. Error bars show the SD across the $10 \mathrm{H} 1$ recordings. The shaded area shows the domain of uncertainty (see Materials and Methods). $c$, Same data as in $b$ but the noise correlation was varied at the "best" temporal resolution. The temporal resolution was $15.625 \mathrm{msec} . d$, Same data as in c but at a temporal resolution used for data analysis of $500 \mathrm{msec}$. e, Mean discrimination performance at $100 \%$ noise correlation and a noise level of $\sigma=16$ as a function of the temporal resolution for four $\mathrm{H} 1 \mathrm{recordings} \mathrm{of} \mathrm{four} \mathrm{different} \mathrm{flies.}$ The error bars represent the SD of performance levels. Shown in gray is the domain of uncertainty, which represents the performance levels that are likely to be the result of internal noise and photon noise but not of the added external noise.

relatively fine time scale, as is the case for uncorrelated noise, but also on a coarser time scale. When the noise was $100 \%$ correlated (i.e., the brightness fluctuations of all 10 dots are identical), reference and test responses could be discriminated with temporal resolutions of up to $1000 \mathrm{msec}$ (Fig. $5 a$, transection b, $b$ ). With the 
finest temporal resolution, the discrimination performance is significantly different from chance level but does not reach the $75 \%$ threshold. However, even with $100 \%$ noise correlation at the maximum noise level $(\sigma=64)$, no effect on the mean spike rate could be observed (only 1 of the 10 cells showed a significant difference in the mean spike rate; $\alpha<5 \%$, Wilcoxon's rank sum test; the mean spike frequency of the corresponding reference responses was $78.6 \mathrm{~Hz}$ with a SD of $1.55 \mathrm{~Hz}$; the test responses had a mean and SD of 80.7 and $2.3 \mathrm{~Hz}$, respectively). At the temporal resolution, which led to the best discrimination performance (15.625 msec) with uncorrelated external noise (compare Fig. 4), we found a weak increase in performance with increasing noise correlation (Fig. 5a, transection c, c). At this temporal resolution, the performance was above threshold for all correlation levels. Again, the responses of some cells could not be distinguished with $>75 \%$ performance. With a coarser temporal resolution (500 msec; Fig. $5 a$, transection d, $d$ ), a more obvious increase in performance could be observed. Although it was below threshold and not clearly different from chance level without noise correlation, a major impact of the external noise was obtained at $100 \%$ noise correlation.

When adding noise to the moving dots with a noise level of $\sigma=16$ and $100 \%$ correlation, the responses to reference and test stimuli are nearly indistinguishable for all temporal resolutions $(n=4$ recorded in four different flies; Fig. 5e). Hence, even for absolutely correlated noise, a noise level, more than four times larger than photon noise, is needed to affect the responses significantly.

\section{Conclusion}

The temporal structure of the $\mathrm{H} 1$ responses to motion stimuli is affected by superimposed noise only if the noise fluctuations cover most of the available brightness range of the monitor screen and, thus, is much larger than photon noise under our experimental conditions. Then, the temporal structure of the responses is affected on a time scale in the range of $\sim 15 \mathrm{msec}$. If the noise added to different dots of the motion stimulus is correlated, the range of temporal resolutions at which changes in the response can be detected is extended. Nevertheless, a high noise level is needed to affect the responses, even when the noise in the input signal is $100 \%$ correlated. Thus, both sets of experiments, using uncorrelated as well as correlated noise superimposed on the input signal, support the conclusion of photon noise being of only minor importance for the reliability of motion vision in $\mathrm{H} 1$.

\section{Discussion}

\section{Comparison with photon noise}

Is the neuronal variability a consequence of the noisy visual input (i.e., photon noise), as has been proposed recently (Borst and Haag, 2001; Lewen et al., 2001), or does it emerge within the nervous system? Whereas these studies applied more indirect methods to tackle this question, with our approach we directly manipulated the input signal. The brightness modulations superimposed on the dots mimicked photon noise and, by varying the strength of the modulations, allowed us to assess the impact of photon noise on the reliability of the $\mathrm{H} 1$ responses.

The overall system noise is approximately equivalent to a noise level of $\sigma>32$ in our stimulus paradigm (compare Fig. 4). How much of the overall noise can be attributed to photon noise, and to noise emerging within the visual system? As estimated in Materials and Methods, photon noise under the conditions of our experiments corresponds to a noise of approximately $\sigma=4$ in our paradigm. Thus, neuronal unreliability, rather than pho- ton noise, seems to be the major noise source that limits the reliability of visual motion computation.

This conclusion needs to be qualified in two respects. First, the estimation of the relevant photon noise is based on the implicit assumption that noise in only those photoreceptors that are stimulated by the moving dots contribute to the response variability of H1. This assumption seems to be justified, in a first approximation, because the stationary background was much darker than the dots and the variability found in fly motion-sensitive neurons during stimulation with stationary dark patterns was much smaller than during motion stimulation (Hengstenberg, 1982; Warzecha, 1994). Second, it was assumed that each motion dot perfectly covers the receptive field of just a single photoreceptor and does not affect neighboring receptors. Because this may frequently not be the case, the photons emitted by a dot may be shared, as a consequence of the hexagonal lattice of ommatidia, by three adjacent photoreceptors. As a consequence, the amount of photons reaching a single receptor and, thus, the SNR of the input signal may be reduced. In the worst case, a single dot is centered above the corner of three neighboring ommatidia. This would reduce the number of photons reaching a single photoreceptor by two-thirds. To meet the worsened SNR caused by light reduction, an added noise with a SD of approximately $\sigma=6.8$ would be needed. Even this noise level is much smaller than the noise level found to influence the $\mathrm{H} 1$ responses. In contrast, in the fly retina, the signals of six photoreceptors sampling the same point in space are averaged by electric coupling of their axons (Laughlin, 1994). This coupling would reduce the variance in the averaged signal by the square root of 6 , because photon noise is independent for each of them.

Moreover, at noise levels of more than twice this noise level, the responses to the reference and to the test stimulus are indistinguishable even when the noise added to all moving dots is completely correlated (Fig. 5e). Because photon noise affecting different photoreceptors is statistically independent, this finding further corroborates our conclusion that under the conditions of our experimental analysis photon noise does not limit the reliability of neural coding. Rather, we can conclude that the variability in the timing of spikes in $\mathrm{H} 1$ is mainly a consequence of noise sources intrinsic to the nervous system. This conclusion is likely to extend to most light levels when the diurnal flies fly around, because our stimulus was much darker than daylight. Under behaviorally relevant light conditions, the number of photon arrivals and, thus, the SNR is much higher.

The conclusion that noise intrinsic to the nervous system limits the reliability of motion information processing is in accordance with experimental data on the peripheral visual system of the fly. Although in completely dark-adapted eyes single photons could be inferred to affect the signal of the $\mathrm{H} 1$ neuron (Lillywhite and Dvorak, 1981), even at moderate light levels that are still much lower than daylight, individual photons only have a small effect on the overall photoreceptor potential, and the synapse between photoreceptor and second-order neurons contribute significantly to the response variability at this processing stage (Laughlin et al., 1987; Juusola et al., 1995; de Ruyter van Steveninck and Laughlin, 1996). Thus, our results are in line with previous findings that suggest that, at least for light levels as used in our experiments and for larger ones, internal noise sources are of more importance than the noise in the visual input (Laughlin et al., 1987; de Ruyter van Steveninck and Laughlin, 1996) but are in contrast with other interpretations (Borst and Haag, 2001; Lewen et al., 2001). 


\section{Comparison with contradictory interpretations}

Lewen et al. (2001) performed methodologically challenging outdoor recordings of $\mathrm{H} 1$ while rotating the whole fly about its vertical body axis in a naturalistic manner. Experiments were performed with the same fly at different times of the day and, thus, at different mean light levels. From noon to half an hour after sunset, the $\mathrm{H} 1$ cell responded to the same motion sequence with a decreasing mean spike rate. The conclusion of photon noise being the limiting factor is based on the finding that the information rate increases with an increasing light level and, thus, with an increasing SNR of the stimulus. However, in other studies, the information rate was shown to be linked approximately linearly to the mean number of spikes, even if spike activity was increased at the same mean light level by changes in stimulus parameters such as velocity or contrast (Schneidman et al., 2000; Borst and Haag, 2001). Hence, the changes in the information rate observed during the course of the day may be interpreted as a consequence of an increasing spike rate rather than SNR of the incoming signals. This suggestion is corroborated by the finding that during the course of the day changes in spike rate are more likely linked to changes in ambient temperature than to changes in light level (Warzecha et al.,1999; Egelhaaf et al., 2001). On this basis, we conclude that the experimental results of Lewen et al. (2001) do not necessarily disagree with our finding that the performance of the H1-neuron is mainly limited by noise sources intrinsic to the nervous system rather than by photon noise.

Borst and Haag (2001) analyzed the amount of information transmitted by $\mathrm{H} 1$ in dependence of the activity level that was altered by visual motion stimulation. The experimental results were compared with predictions that were drawn from a model $\mathrm{H} 1$ that receives input from eight correlation type motion detectors and transforms the resulting graded membrane potential changes into spike trains using a Hodgkin-Huxley-type spike generator. The input signal of the model was additionally corrupted by brightness modulations mimicking photon noise that were adjusted to match experimentally found response variability. Because the performance of both real and model $\mathrm{H} 1$ were similar and no other noise but photon noise was introduced into the system, it was concluded that photon noise is the dominant noise source of the fly's visual motion pathway. Given that the model H1 cell was driven by only eight motion detectors, their gain had to be sufficiently high to drive the cell to a realistic activity level. However, under the stimulus conditions of the experiments of Borst and Haag (2001), the H1 cell receives input from hundreds of motion-sensitive elements that contribute to the overall response. Hence, the individual input channels are likely to have contributed much less to the variability of the $\mathrm{H} 1$ neuron than was assumed in the corresponding model simulations. Thus, these results do not seem to contradict our conclusion that the noise level found in $\mathrm{H} 1$ is mainly determined by sources intrinsic to the nervous system rather than by photon noise.

\section{References}

Barberini CL, Horwitz GD, Newsome WT (2000) A comparison of spiking statistics in motion sensing neurons of flies and monkeys. In: Computational, neural and ecological constraints of visual motion processing (Zanker JM, Zeil J, eds), pp 307-320. Heidelberg: Springer.

Berry MJ, Warland DK, Meister M (1997) The structure and precision of retinal spike trains. Proc Natl Acad Sci USA 94:5411-5416.

Borst A, Haag J (2001) Effects of mean firing on neural information rate. J Comput Neurosci 10:213-221.

Borst A, Haag J (2002) Neural networks in the cockpit of the fly. J Comp Physiol [A] 188:419-437.
Bouman MA, van de Grind MA, Zuidema P (1985) Quantum fluctuations in vision. In: Progress in Optics, Vol 22 (Wolf E, ed), pp 79-144. Amsterdam: North Holland.

Britten KH, Shadlen MN, Newsome WT, Movshon JA (1993) Responses of neurons in macaque MT to stochastic motion signals. Vis Neurosci 10:1157-1169.

de Ruyter van Steveninck R (1986) Real-time performance of a movementsensitive neuron in the blowfly visual system. PhD thesis, Groningen University.

de Ruyter van Steveninck R, Bialek W (1995) Reliability and statistical efficiency of a blowfly movement-sensitive neuron. Philos Trans R Soc Lond B Biol Sci 348:321-340.

de Ruyter van Steveninck R, Laughlin SB (1996) The rate of information transfer at graded-potential synapses. Nature 379:642-645.

de Ruyter van Steveninck R, Borst A, Bialek W (2001) Real-time encoding of motion: answerable questions and questionable answers from the fly's visual system. In: Motion vision (Zanker JM, Zeil J, eds), pp 279-306. Berlin, Heidelberg, New York: Springer.

Egelhaaf M, Warzecha AK (1999) Encoding of motion in real time by the fly visual system. Curr Opin Neurobiol 9:454-460.

Egelhaaf M, Grewe J, Kern R, Warzecha AK (2001) Outdoor performance of a motion-sensitive neuron in the blowfly. Vision Res 41:3627-3637.

Egelhaaf M, Kern R, Krapp HG, Kretzberg J, Kurtz R, Warzecha AK (2002) Neural encoding of behaviourally relevant visual-motion information in the fly. Trends Neurosci 25:96-102.

Franceschini N (1975) Sampling of the visual environment by the compound eye of the fly: fundamentals and applications. In: Photoreceptor optics (Snyder AW, Menzel R, eds), pp 98-125. Berlin, Heidelberg, New York: Springer.

Götz KG (1965) Die optischen Übertragungseigenschaften der Komplexaugen von Drosophila. Kybernetik 2:215-221.

Haag J, Borst A (2002) Dendro-dendritic interactions between motionsensitive large-field neurons in the fly. J Neurosci 22:3227-3233.

Hardie RC (1979) Electrophysiological analysis of the fly retina. I. Comparative properties of R1-6 and R7 and R8. J Comp Physiol [A] 129:19-33.

Hausen K (1976) Functional characterization and anatomical identification of motion sensitive neurons in the lobula plate of the blowfly Calliphora erythrocephala. Z Naturforsch 31c: 629-633.

Hausen K (1982) Motion sensitive interneurons in the optomotor system of the fly. I. The horizontal cells: structure and signals. Biol Cybern 45:143-156.

Hengstenberg R (1982) Common visual response properties of giant vertical cells in the lobula plate of the blowfly Calliphora. J Comp Physiol 149:179-193

Johnston D, Wu S (1995) Foundations of cellular neurophysiology. Cambridge, MA: MIT.

Juusola M, Uusitalo RO, Weckström M (1995) Transfer of graded potentials at the photoreceptor-interneuron synapse. J Gen Physiol 105:117-148.

Kara P, Reinagel P, Reid RC (2000) Low response variability in simultaneously recorded retinal, thalamic, and cortical neurons. Neuron 27:635-646.

Kretzberg J, Warzecha AK, Egelhaaf M (2001) Neural coding with graded membrane potential changes and spikes. J Comput Neurosci 11:153-164.

Laughlin SB (1994) Matching coding, circuits, cells, and moleculees to signals: general principals of retinal design in the fly's eye. Prog Retinal Eye Res 13:165-196.

Laughlin SB, Howard J, Blakeslee B (1987) Synaptic limitations to contrast coding in the retina of the blowfly Calliphora. Proc R Soc Lond B Biol Sci 231:437-467.

Levine MW, Zimmerman RP, Carrion-Carire V (1988) Variability in responses of retinal ganglion cells. J Opt Soc Am A 5:593-597.

Lewen GD, Bialek W, de Ruyter van Steveninck R (2001) Neural coding of naturalistic motion stimuli. Network 12:317-329.

Lillywhite PG, Dvorak DR (1981) Responses to single photons in a fly optomotor neurone. Vision Res 21:279-290.

Pelli DG (1990) The quantum efficiency of vision. In: Vision-coding and efficiency (Blakemore C, ed), pp 1-24. Cambridge: Cambridge UP.

Reichardt W (1965) Quantum sensitivity of light receptors in the compound eye of the fly Musca. Cold Spring Harbor Symp Quant Biol 30:505515 .

Rodieck RW (1998) The first steps in seeing. Sunderland, MA: Sinauer. 
Schneidman E, Brenner N, Tishby N, de Ruyter van Steveninck R, Bialek W (2001) Universality and individuality in a neural code. In: Advances in neural information processing, Vol 13 (Leem TK, Dietterick TG, Tresp V, eds), pp. 151-165. Cambridge, MA: MIT.

Smakman JGJ, Hateren JHV, Stavenga DG (1984) Angular sensitivity of blowfly photoreceptors: intracellular measurements and wave-optical predictions. J Comp Physiol [A] 155:239-247.

Tolhurst DJ, Movshon JA, Dean AF (1983) The statistical reliability of signals in single neurons in cat and monkey visual cortex. Vision Res 23:775-785.

van Hateren JH (1984) Waveguide theory applied to optically measured angular sensitivities of fly photoreceptors. J Comp Physiol [A] 154:761-771.

Victor JD, Purpura KP (1997) Metric-space analysis of spike trains: theory, algorithms and application. Network Comput Neural Syst 8:127-164.

Vogels R, Spileers W, Orban GA (1989) The response variability of striate cortical neurons in the behaving monkey. Exp Brain Res 77:432-436.

Warzecha AK (1994) Reliability of neuronal information processing in the motion pathway of the blowflies Calliphora erythrocephala and Lucilia cuprina. PhD thesis, Universität Tübingen.
Warzecha AK, Egelhaaf M (1999) Variability in spike trains during constant and dynamic stimulation. Science 283:1927-1930.

Warzecha AK, Egelhaaf M (2001) Neuronal encoding of visual motion in real-time. In: Motion vision-computational, neural, and ecological constraints (Zanker JM, Zeil J, eds), pp 239-277. Berlin, Heidelberg, NewYork: Springer.

Warzecha AK, Egelhaaf M, Borst A (1993) Neural circuit tuning fly visual interneurons to motion of small objects. I. Dissection of the circuit by pharmacological and photoinactivation techniques. J Neurophysiol 69:329-339.

Warzecha AK, Horstmann W, Egelhaaf M (1999) Temperaturedependence of neuronal performance in the motion pathway of the blowfly Calliphora erythrocephala. J Exp Biol 202:3161-3170.

Warzecha AK, Kretzberg J, Egelhaaf M (2000) Reliability of a fly motionsensitive neuron depends on stimulus parameters. J Neurosci 20:8886-8896.

Zohary E, Shadlen MN, Newsome WT (1994) Correlated neuronal discharge rate and its implications for psychophysical performance. Nature 370:140-143. 